\title{
FORECASTING THE WIMAX TRAFFIC VIA MODIFIED ARTIFICIAL NEURAL NETWORK MODELS
}

\author{
Daw Abdulsalam Ali Daw ${ }^{1 *}$, Kamaruzzaman Bin Seman ${ }^{1}$, Madihah Bint Mohd \\ Saudi $^{2}$ \\ ${ }^{1 *}$ Corresponding author: Daw Abdulsalam Ali Daw \\ Faculty of science and Technology, University Sains Islam Malaysia \\ Malaysia
}

\begin{abstract}
This paper attempts to present a new approach of forecasting the WiMAX traffic by exploiting Artificial Neural Networks (ANN). To develop the model, actual data is gathered from the LibyaMax network that spans the duration of 180 days in total. Traffic data is separated into three cases based on the base stations involved $(A, B$ and $A B)$. The model implements traffic prediction by emphasizing on the maximum and minimum number of online user whereby two different learning algorithms are tested upon. to find the optimal one. Overall, the experimentation shows promising results of which the most severe error of prediction is not more than 0.0014. This indicates the feasibility of making accurate forecasting of both daily and weekly traffic of the WiMAX network based solely on the maximum and minimum number of users online.
\end{abstract}

\section{KEYWORDS}

WIMAX traffic, ANN model, Forecasting System

\section{INTRODUCTION}

Being able to predict the traffic of a particular WiMAX network is rather crucial in analyzing its performance. It bears various applications in reality. For instance, to enable better network management and admission. Furthermore, traffic forecasting plays a vital role in ensuring that the quality of service is maintained at the necessary level. In this respect, it is an obligatory process that must be adhered upon. Prediction works closely with decision making in network design. By predicting the impact of changing a certain part of the network architecture, more effective decision can be made regarding the best viable structure in a particular situation. As such, it is critical to capture the behavior of traffic accurately. To this end, many models have been proposed and revised for the purpose of simulating and understanding the inherent dynamics of a network. The main idea of traffic prediction is to forecast the possible future traffic by analyzing a series of recorded traffic in the past. Traffic data that is gathered through time must be tested rigorously to validate its usefulness in developing the predictive model. Other factors such as the actual prediction phase, prediction malfunction and computational cost must also be taken into serious consideration in constructing a versatile forecasting model.

DOI : 10.5121/ijaia.2014.5503 
One of the most challenging issues to be addressed in data mining research is the exploration of time series data [1]. Several prediction methods are considered in deriving the most appropriate summary and conclusion. Based on commonality, the procedure of these methods in time series is assumed to be linear. However, they can also be non-linear[2] of which linear approaches will no longer apply. Now, if the approach is developed on the basis of Box-Jenkins method, then it would be advisable to construct the time series model in a sequence of steps, before discovering the ideal model. A series of alternative models can also be found by using structural state space methods, which allows the estimation of stationary, trend, seasonal and cyclical data. Compared to other methods, they capture the summation of separate components to enable analysis.

Among all the available models, Artificial Neural Network (ANN) displays an affinity to give superior results [3][4][5]. Compared with ARIMA or fractional ARIMA predictors [6], the performance of ANN is rather balanced when contrated with its computational intricacy. This research accentuates the impact of using ANN in traffic forecasting for WiMAX network whereby the advantage [7] is properly demonstrated in comparison with standard rule-based systems. Apart from that, others [8][9][10] have also recommended the use of Time Delayed Neural Network (TDNN). However, this particular approach will not be included here.

To generate the traffic, an integration of the entire network system is required. This includes the traffic source [11] as well as the prediction technique that provides future energy prices, specifically one day earlier. Here, Wavelet Transform offers a highly beneficial pre-processing approach for forecasting data that can enhance the performance of prediction strategies as a whole. The proposed forecasting model in this research deviates from the decomposition of time series in the wavelet domain [12].

Instead, it employs ANN in the transform domain by exploiting the Alcatel-Lucent study [13]. In addition, the impact of different ANN configurations on prediction performance will also be examined in the end.

The following section narrates the forecasting structure as well as the theoretical consideration with regard to data collection. A brief explanation on ANN and its theory are also included, along with its configuration and implementation. In section three, the result and the discussion are presented. Finally, the conclusion and future work are covered in the last section.

\section{METHODOLOGY}

\section{A. Data Collection}

Data for this research is gathered from the WiMAX traffic of Tripoli, the capital city of Libya. It is required in the construction of the ANN based model for the WiMAX traffic forecasting analysis. The data consist of two collection of scenarios. The first collection (Figure 1(a,b), $2(a, b), 3(a, b))$ entails the daily data during the 180 days. On the other hand, the second collection (Figure 4(a,b), 5(a,b), 6(a,b)) covers the weekly data within the 180 days duration. Each collection includes the maximum and minimum number of user-A, user-B and user- $\mathrm{AB}$ as well as their WiMAX traffic of MIMO-A, MIMO-B and MIMO-AB. 
International Journal of Artificial Intelligence \& Applications (IJAIA), Vol. 5, No. 5, September 2014

Now, for the daily traffic, the maximum number of online users is generally between 25 to 80 people. On the contrary, the minimum number of online users is between 20 to 75 people. In terms of traffic, MIMO-A user shows a range of traffic between $2 \mathrm{e} 8$ and $10 \mathrm{e} 8$ byte. MIMO-B user however, shows a degree of traffic between $0.5 \mathrm{e} 8$ to $5.5 \mathrm{e} 8$ byte.

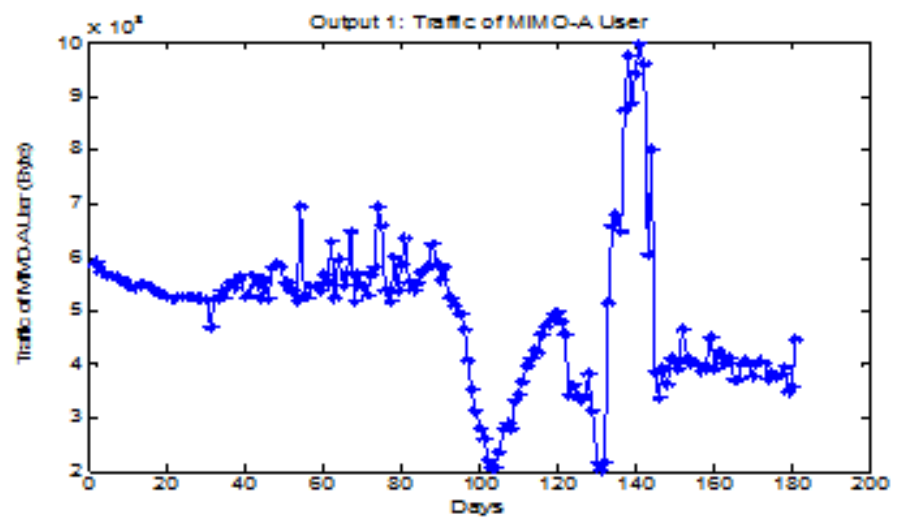

Figure 1(a). Daily data input Max number online of user-A,

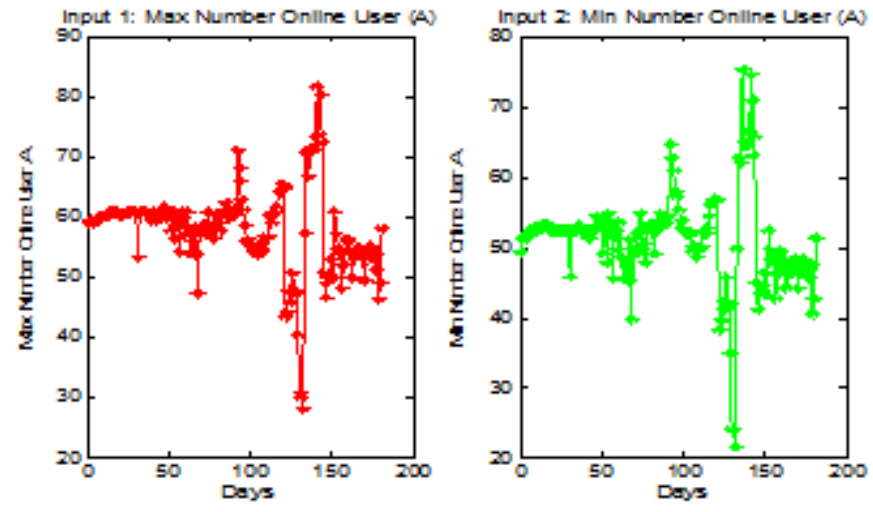

Figure 1(b). Daily data Output Min number online user A and traffic of MIMO-user A 
International Journal of Artificial Intelligence \& Applications (IJAIA), Vol. 5, No. 5, September 2014
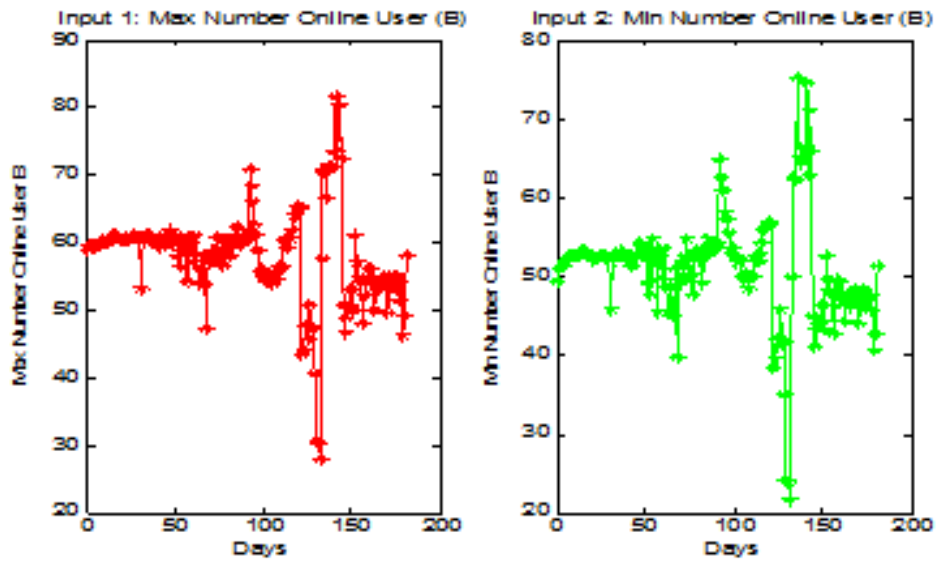

Figure 2 (a). Daily data input Max number online of user-B,
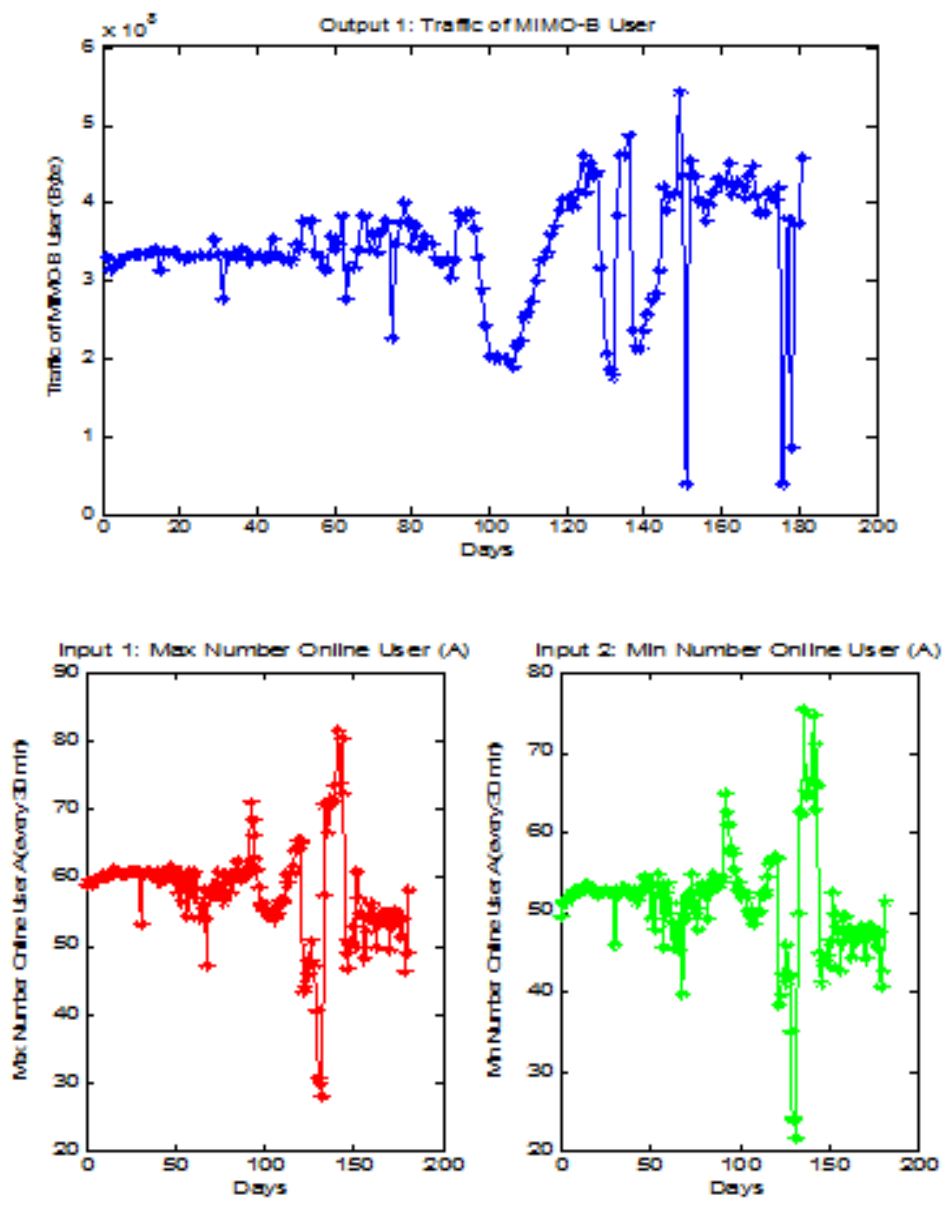

Figure 3 (a). Daily data input Max number online of user-AB, 
International Journal of Artificial Intelligence \& Applications (IJAIA), Vol. 5, No. 5, September 2014

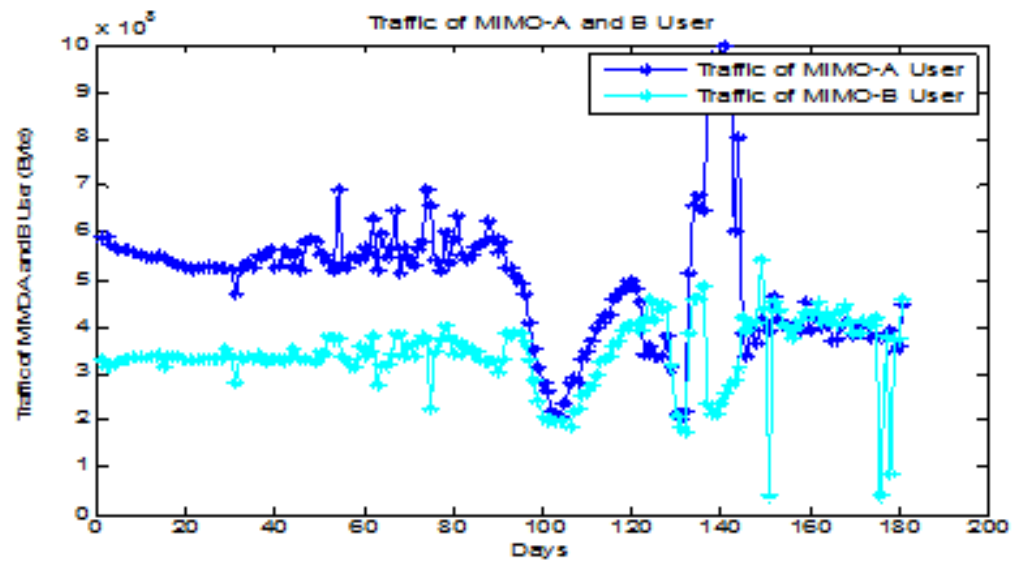

Figure 3(b). Daily data Output Min number online user A and traffic of MIMO-user AB.

For the weekly traffic, the maximum number of online users is between 40 to 70 people while the minimum number lies between 33 and 66 people. The range of traffic for weekly use is approximately between $2.4 \mathrm{e} 8$ to $8.1 \mathrm{e} 8$ byte for MIMO-A user and 2.0e 8 to $4.3 \mathrm{e} 8$ byte for MIMO$\mathrm{B}$ user. This indicates that the former user displays a higher level of weekly traffic as compared to the latter.
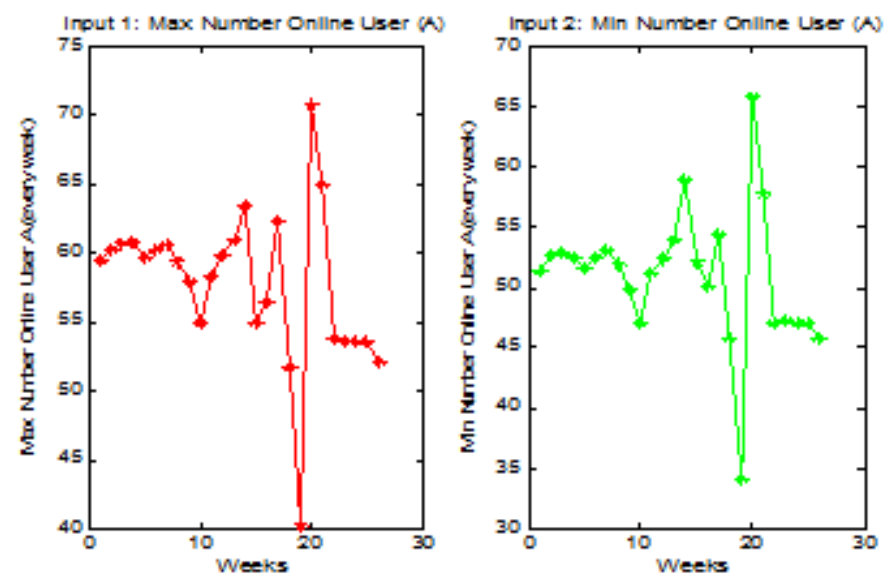

Figure 4 (a). Weekly data input Max number online of user-A, 
International Journal of Artificial Intelligence \& Applications (IJAIA), Vol. 5, No. 5, September 2014

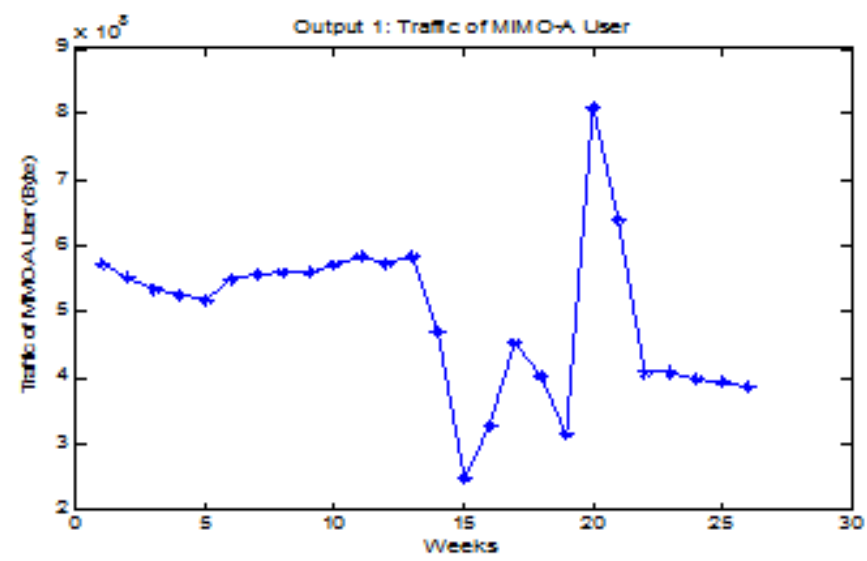

Figure 4 (b). Weekly data Output Min number online user A and traffic of MIMO-user A
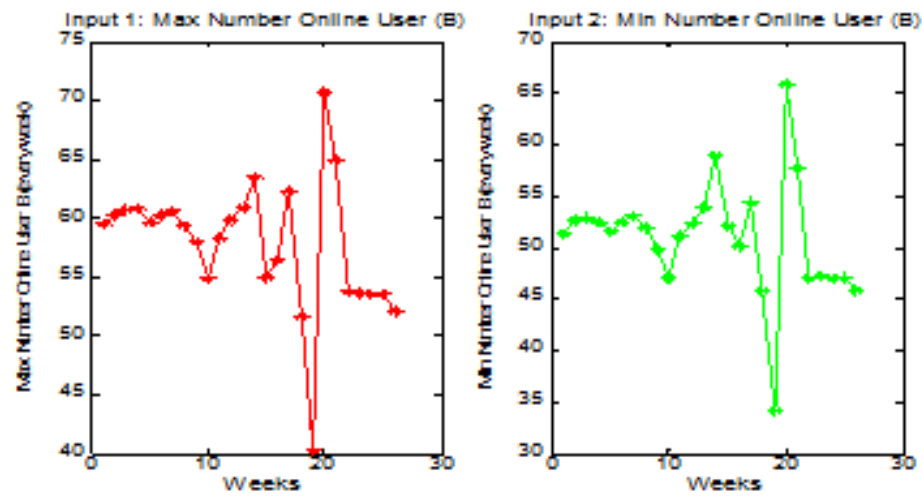

Figure 5 (a). Weekly data input Max number online of user-B,

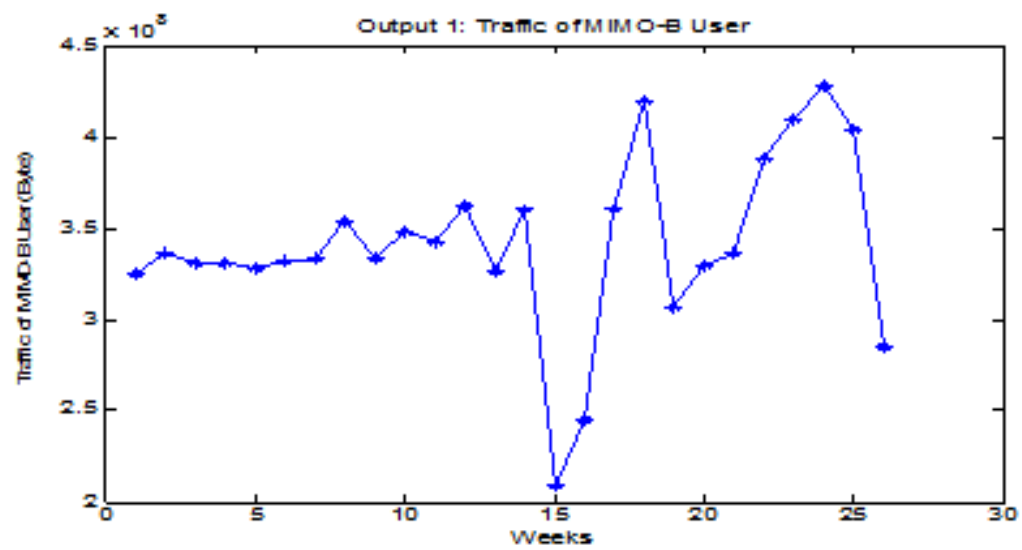

Figure 5 (b). Weekly data Output Min number online user A and traffic of MIMO-user B. 

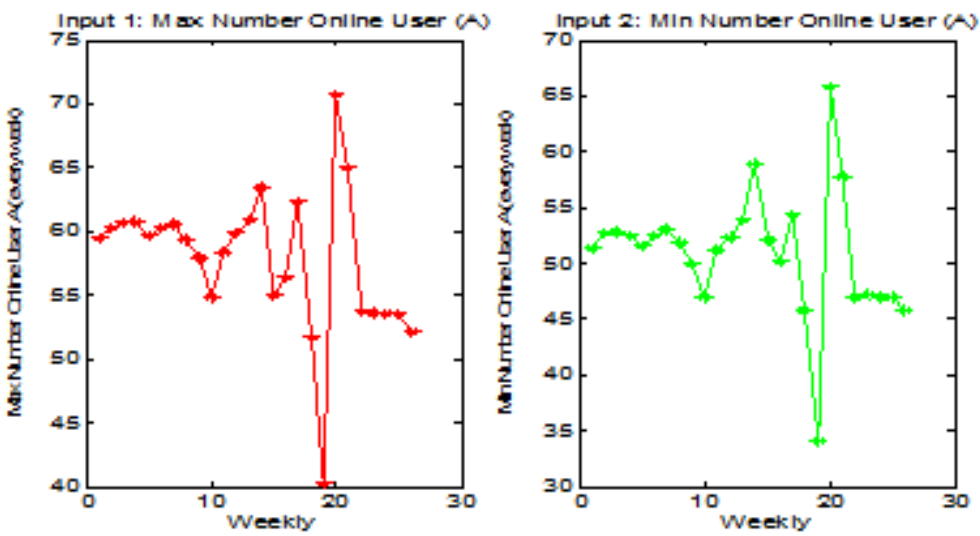

Figure 6 (a). Weekly data input Max number online of user-AB,

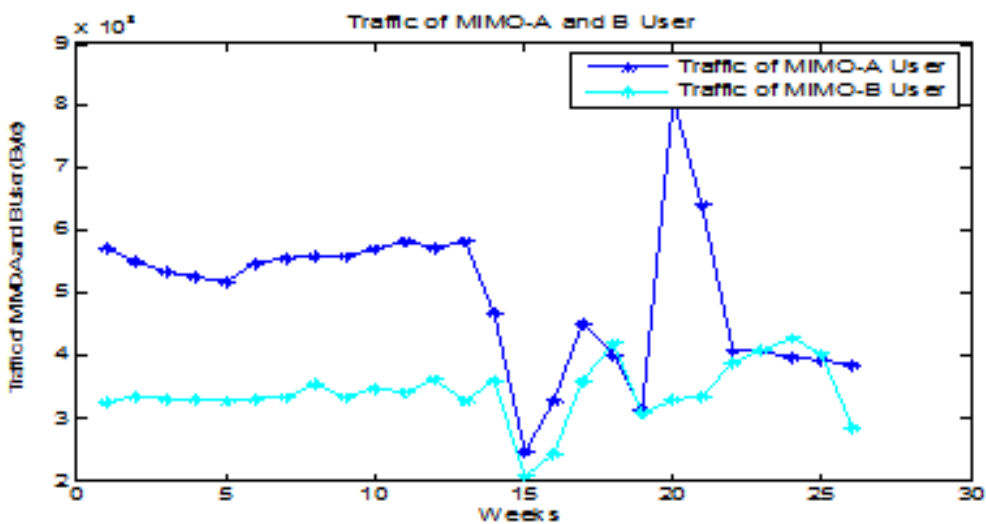

Figure 6 (b). Weekly data Output Min number online user A and traffic of MIMO-user AB.

\section{B. ANN based Implementation of WIMAX Traffic Forecasting}

To previse the WiMAX traffic using the ANN model, the multilayered feed forward perceptron is exploited. Three sub-models are designed for this purpose whereby each involves network architecture with input and output layers, as well as the hidden layers in between. The structure of the hidden layer is shown in Figure 7. The data for the input layer consists of the maximum and minimum number of online users. However, data for the output layer involves the traffic of MIMO-A, MIMO-B and MIMO-AB. As such, three configuration patterns or models of training are employed. Each configuration works on two time frames, which are daily and weekly. To explain the idea in more detail, consider the following:

- Case (1): WIMAX traffic of MIMO-A users

Here, the effort of estimating the WiMAX Traffic comes from MIMO-A users. This is done using the daily and weekly recorded data, which include the maximum and the 
International Journal of Artificial Intelligence \& Applications (IJAIA), Vol. 5, No. 5, September 2014

minimum number of online users only. Thus, the traffic from MIMO-A users of daily and weekly data are given as:

$$
\begin{aligned}
& T_{\text {Daily (A) }}=f_{A}\left(X_{\text {user (Max) }}, X_{\text {user (Min) }}\right) \\
& T_{\text {Weekly (A) }}=f_{A}\left(X_{\text {user (Max) }}, X_{\text {user (Min) }}\right)
\end{aligned}
$$

- Case (2): WIMAX traffic of MIMO-B users

The prediction of daily and weekly traffic for MIMO-B user are attained only by considering the maximum and the minimum number of its online users. In effect, so the traffic from MIMO-B users for both time frames, daily and weekly, are :

$$
\begin{aligned}
& T_{\text {Daily }(B)}=f_{B}\left(X_{u s e r(\operatorname{Max})}, X_{u s e r(\operatorname{Min})}\right) \\
& T_{\text {Weekly }(B)}=f_{B}\left(X_{u s e r(\operatorname{Max})}, X_{u s e r(\operatorname{Min})}\right)
\end{aligned}
$$

\section{- Case (3): WIMAX traffic of MIMO-AB users}

For the final scenario, both aforementioned cases are combined. The prediction of WiMAX traffic for MIMO-AB users from the aspect of daily and weekly, are derived by utilizing the daily and weekly data recorded :

$$
\begin{aligned}
& T_{\text {Daily }(A B)}=f_{A B}\left(X_{\text {user }(\operatorname{Max})}, X_{\text {user }(\operatorname{Min})}\right) \\
& T_{\text {Weekly }(A B)}=f_{A B}\left(X_{u \operatorname{ser}(\operatorname{Max})}, X_{u \operatorname{user}(\operatorname{Min})}\right)
\end{aligned}
$$

Here, $\left.{ }^{T_{D a i l y}(A, B, A B}\right)$ represents the daily WiMAX traffic from MIMO-A, MIMO-B and MIMO-AB users (byte) respectively while $T_{\text {Weekly }(A, B, A B)}$ corresponds to the weekly traffic. $\left(X_{\text {user }(\operatorname{Max})}\right)$, $\left(X_{\text {user(Min) }}\right)$ represent the maximum and the minimum number of online (Max-online) and (Minonline) respectively, $f_{A, B, A B}$ is the function model depend on the architecture of the neural network as indicated in Figure 7

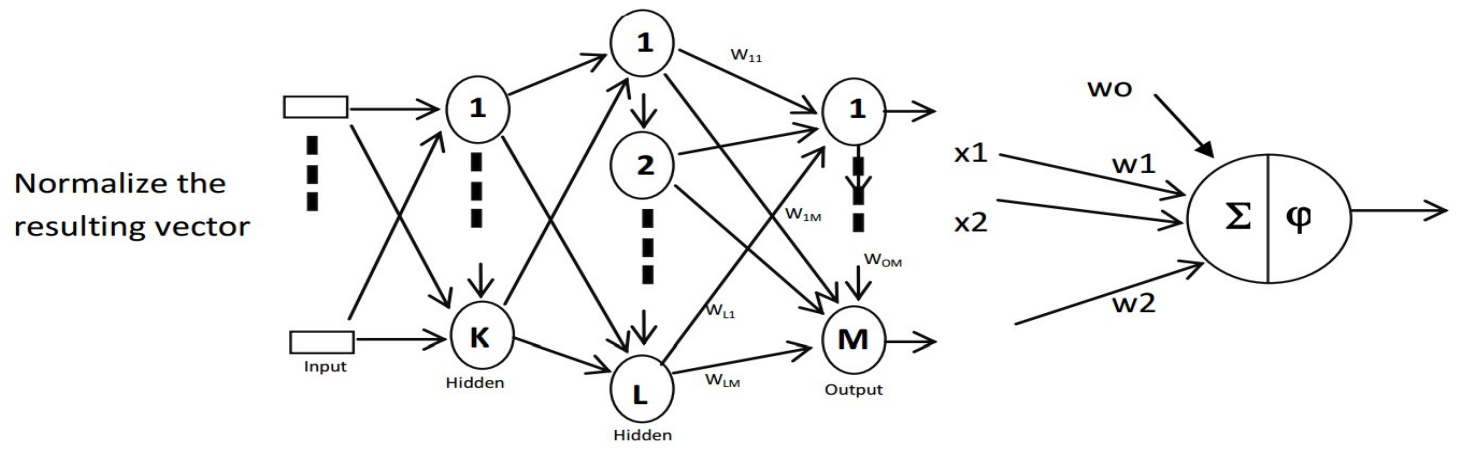

Figure 7. General ANN configuration patterns 
The usage of all the models enables the estimation of traffic in terms of the traffic flow index (output). This provides a prediction on the traffic flow index of the WiMAX network on a daily and weekly basis. Daily and weekly prediction differ from the aspect of overall duration whereby the former is more interested on the short term analysis of the traffic whereby the latter gives a somewhat mid term portrayal of traffic as a whole. Working together, both predictions can assist the process of analyzing the network traffic more accurately.

\section{Training Process}

To perform the training, the back propagation method is employed whereby neurons are trained and adjusted according to the error, with the synaptic weights appropriately modified. The output of the approach depends on the variability of the input. Essentially, the algorithm is supervised and iterated for multilayer feed forward nets, specifically tailored with nonlinear sigmoidal threshold units, defined by the following equation:

$$
f(x)=\frac{1}{\left(1+e^{-x}\right)}
$$

The characteristic of the problem is reflected in the set of input-output vectors within the modeling stage where $2 / 3$ of the entire data is used as training data. Output is gathered and then compared with the one from the training set. The difference detected between the experimental and desired output will then be utilized to modify the connection weights. Computationally, this is done to minimize error, such that it will reach a stipulated level of tolerance.

At the accepted tolerance level, the connection weights are maintained and used to make decision. The training halts when the mean average error between the measured output and desired output remains unchanged after a number of trials. Consequently, the output attained by this procedure will then be contrasted against the targeted value. This is achieved by measuring the error function, which is defined by the average of square difference between the output of each neuron in the output layer and the actual desired output.

This process is conducted on both of the datasets - training and testing. For the training, the parameters are set according to the ones below:

$\begin{array}{ll}\text { Epochs of training } & : 10 \mathrm{e} 4 \\ \text { Training goal } & : 10-3 \\ \text { Momentum constant } & : 0.92 \\ \text { Number of neurons } & :\left[\begin{array}{lll}20 & 10 & 1\end{array}\right] .\end{array}$

The maximum time of training, however, is not set at a particular value because it depends on the learning algorithm. Consistency of the model is achieved by normalizing the input and output data within the range of $(0,1)$. They are returned to the original values after the simulated is completed by employing the following formula:

$$
x^{\prime}=\frac{x-x_{\text {Min }}}{\left(x_{\max }-x_{\text {Min }}\right)}
$$


Here, $(x)$ denotes the inputs/outputs of the network and the normalized version is written as $\left(x^{\prime}\right)$. Normalized values are determined by the maximum and minimum value of the input and output. To note, the value of normalized input or output is 1 when the input or output is $x_{\max }$, and the value of normalized input or output is 0 when the input or output is $x_{\text {Min }}$.

In designing the ANN, the activation function employs the sigmoid function. On the other hand, the "Tansig" transfer function is used in the hidden layer while the "Purelin" transfer function is deployed in the output layer. The "Tansig" transfer function is:

$$
\tan \operatorname{sig}(x)=\frac{2}{1+e^{(-2 x)}}-1
$$

\section{ANN Models Error Analysis (The Proposed)}

To train, validate and test the feed-forward back-propagation neural network, the data gathered from LibyaMax network is used. Later, it is employed to estimate the WIMAX traffic such that the accuracy can be ascertained in the end. This is crucial in verifying the performance of the prediction model whereby the predicted value generated by the model is compared with the actual data obtained. The stability of the model can then be found by evaluating the difference via the calculation of the statistical error, or more specifically, the mean square error (MSE) that exploits the following formula:

$$
M S E=\frac{1}{N} \sum_{i=1}^{N}\left(X_{M}-X_{E}\right)^{2}
$$

$$
\begin{array}{ll}
\mathrm{N} & \text { number of input-output pairs } \\
\mathrm{XM} & \text { desired value } \\
\mathrm{XE} & \text { estimated value }
\end{array}
$$

Further statistical analysis will give rise to a parameter known as the model efficiency $\left(M_{e f f}\right)$ for the ANN prediction results. It is defined as:

$$
M_{\text {eff }}=1-\left(\frac{M S E}{V a r}\right)
$$

Discriminating the best model from the rest can be done by observing the extent of errors that occurred. Statistically speaking, the model having the lowest error (MSE) is deemed as the best one.

\section{RESULT AND DISCUSSIONS}

There are two set of results to be discussed in this particular section. The first set is the daily WiMAX traffic for the Tripoli city and the second one is the weekly WiMAX traffic. For the ANN model, three types of learning algorithms are implemented. They are (a) TrainLM and (b) TrainSCG. The predictive performance of the model is then measured by examining the error (MSE) and Model efficiency (Eff) indicators. Details of the discussion is covered below : 
International Journal of Artificial Intelligence \& Applications (IJAIA), Vol. 5, No. 5, September 2014

\section{Modeling results: daily WiMAX traffic of MIMO-A users}

The result of the experimentation for the daily WiMAX traffic of MIMO-A users is summarized in Table I while the graphical description is given in Figure 8. Overall, the results show remarkable accuracy when plotted against the measured data.

Table I Cross-Validation Report Of Ann Modeling Results Of Wimax Traffic Of Mimo-User A

\begin{tabular}{|l|c|c|c|c|}
\hline \multirow{2}{*}[\begin{array}{l|c|c|}{20}&{10}&{1}\end{array}]{} & \multicolumn{4}{|c|}{$T_{\text {Daily (A) }}=f_{A}\left(X_{\text {user (Max })}, X_{\text {user (Min })}\right)$} \\
\cline { 2 - 5 } & Training & Testing \\
\hline Algorithms & MSE & $\mathrm{M}_{\mathrm{eff}}$ & $\mathrm{R}$ & MSE \\
\hline TrainLM & 0.000099 & 0.9464 & 0.97271 & $\mathbf{0 . 0 0 1 0}$ \\
\hline TrainSCG & 0.001033 & 0.9447 & 0.97166 & 0.0010 \\
\hline
\end{tabular}

From Table I, the average efficiency of the model can be calculated, which is found to be 0.9456 . Among the learning algorithms, TrainLM shows the best performance with a competitive error or MSE (0.0010) and highest efficiency (0.9464). The worst performance is exhibited by TrainSCG with similar MSE (0.0010) and lowest efficiency (0.9447).
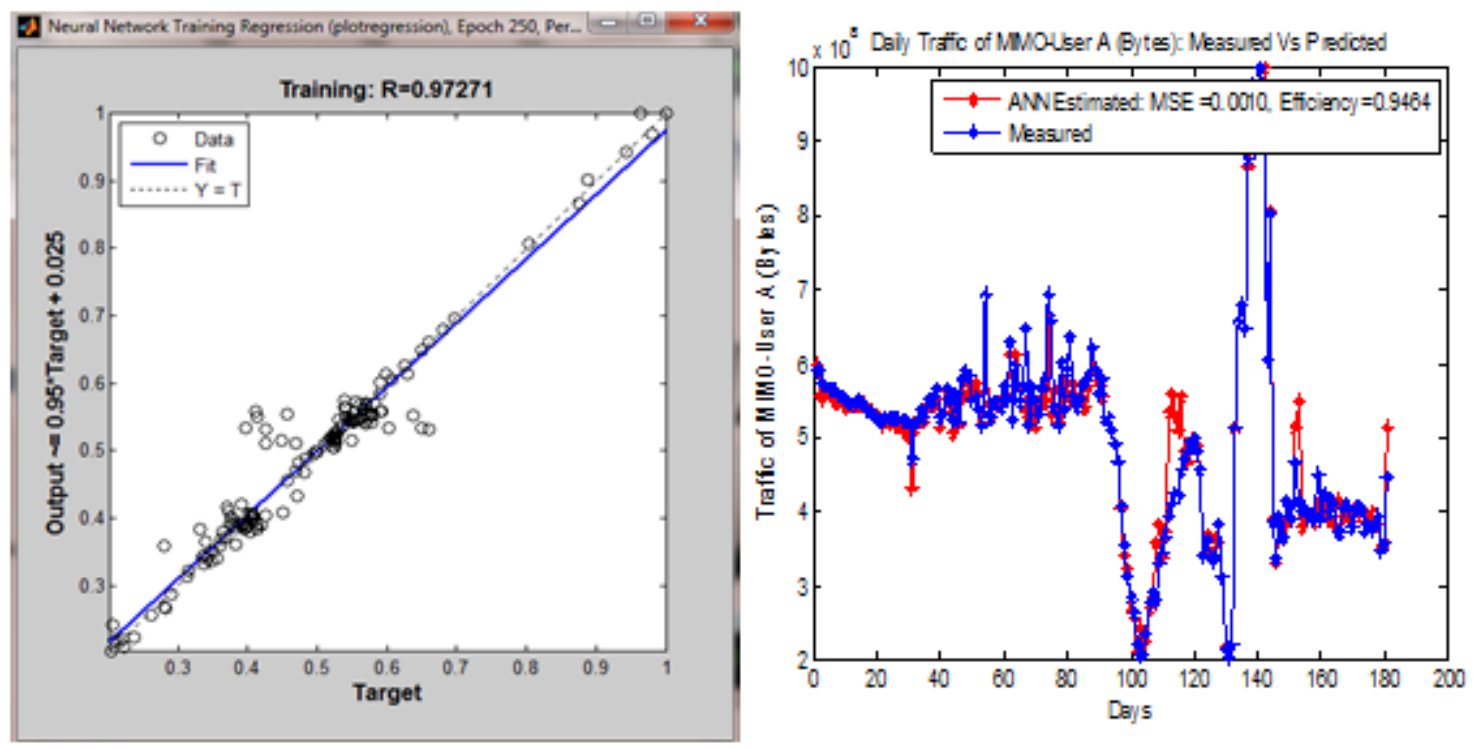

Figure 8. Results of modeling the daily Wimax traffic of MIMO-A users with (a) trainLM 

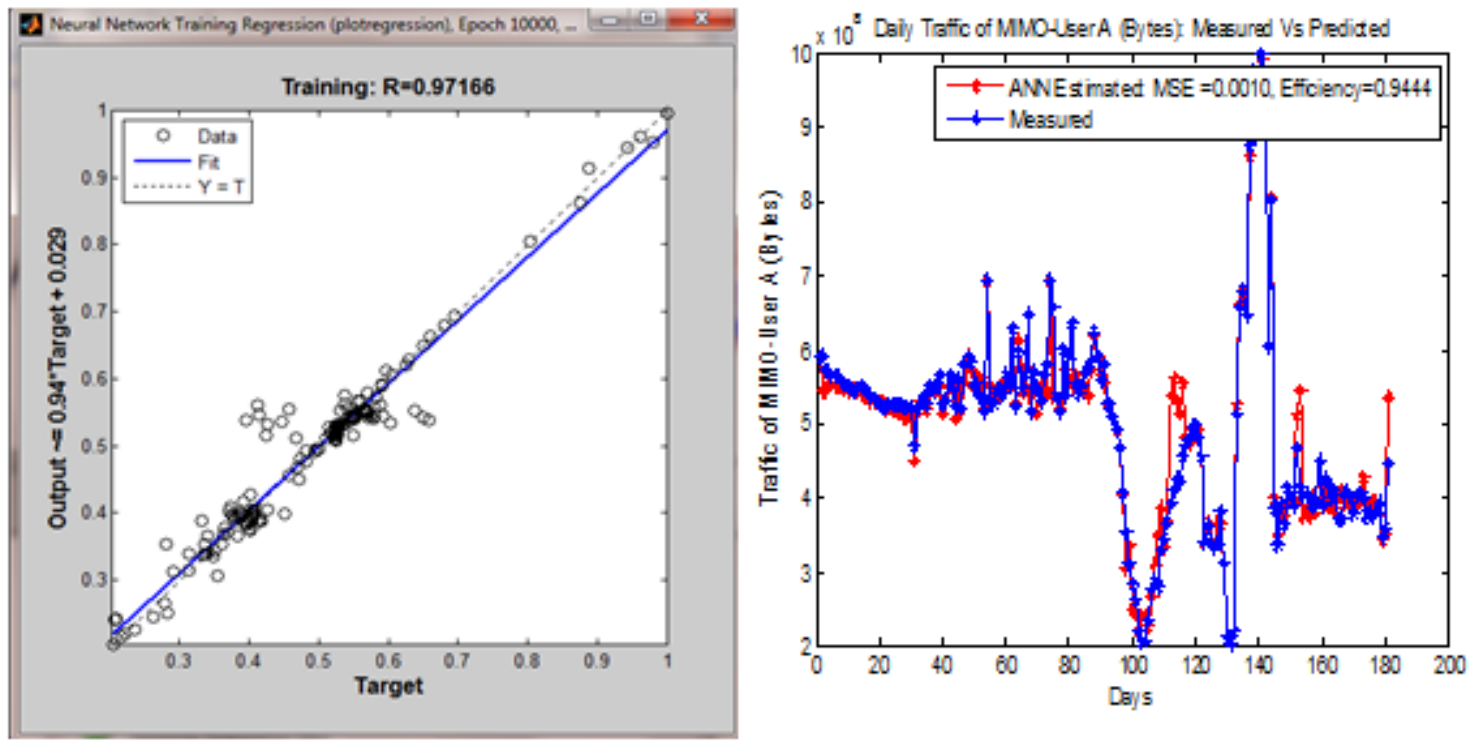

Figure 8. Results of modeling the daily Wimax traffic of MIMO-A users with (b) trainSCG

\section{Modeling results: daily WIMAX traffic of MIMO-B users}

The experimental result for the daily WiMAX traffic of MIMO-B users is given in Table II. Its corresponding graphical depiction is shown in Figure 9.

Table Ii Cross-Validation Report Of Ann Modeling Results Of Daily Wimax Traffic Of Mimo-User B

\begin{tabular}{|l|c|c|c|c|}
\hline \multirow{2}{*}[20101]{} & \multicolumn{4}{|c|}{$T_{\text {Daily }(B)}=f_{B}\left(X_{\text {user }(\operatorname{Max})}, X_{\text {user }(\operatorname{Min})}\right)$} \\
\cline { 2 - 5 } & \multicolumn{3}{|c|}{ Training } & Testing \\
\hline Algorithms & MSE & $\mathrm{M}_{\mathrm{eff}}$ & $\mathrm{R}$ & MSE \\
\hline TrainLM & 0.0009974 & 0.9473 & 0.97316 & $\mathbf{0 . 0 0 1 0}$ \\
\hline TrainSCG & 0.001027 & 0.9407 & 0.89443 & 0.0038 \\
\hline
\end{tabular}

Again, it is discovered here that the best learning algorithm is trainLM with the lowest error MSE (0.0010) and highest efficiency (0.9473). TrainSCG consistently shows the worst performance with the highest MSE (0.0038) and lowest efficiency (0.9407).

The performance of TrainLM in forecasting the daily WiMAX

traffic of MIMO-A users suggests the suitability of this particular learning algorithm in handling traffic data that is specific to this context. The limitation of ANN however, is its inability to explain why certain algorithm is better than another. 

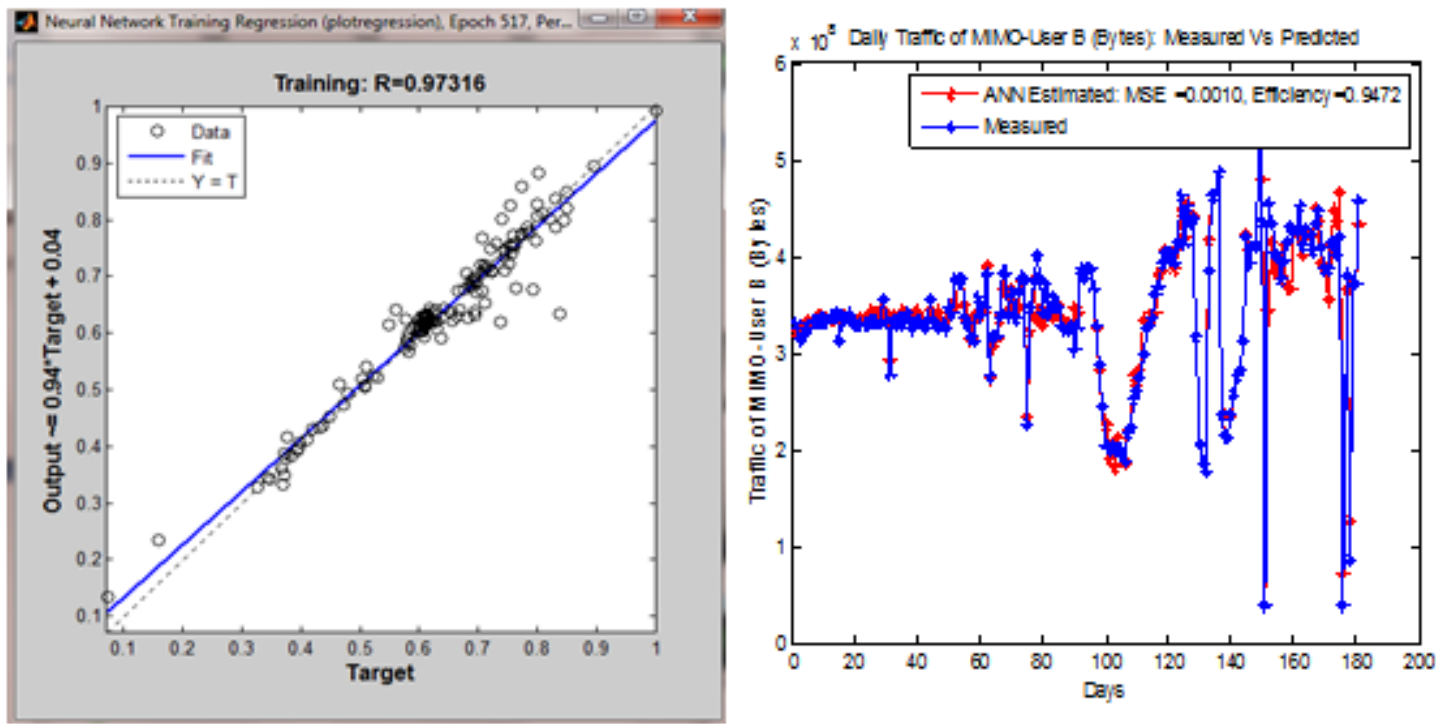

Figure 9. Results of modeling the daily Wimax traffic of MIMO-B users with (a) trainLM
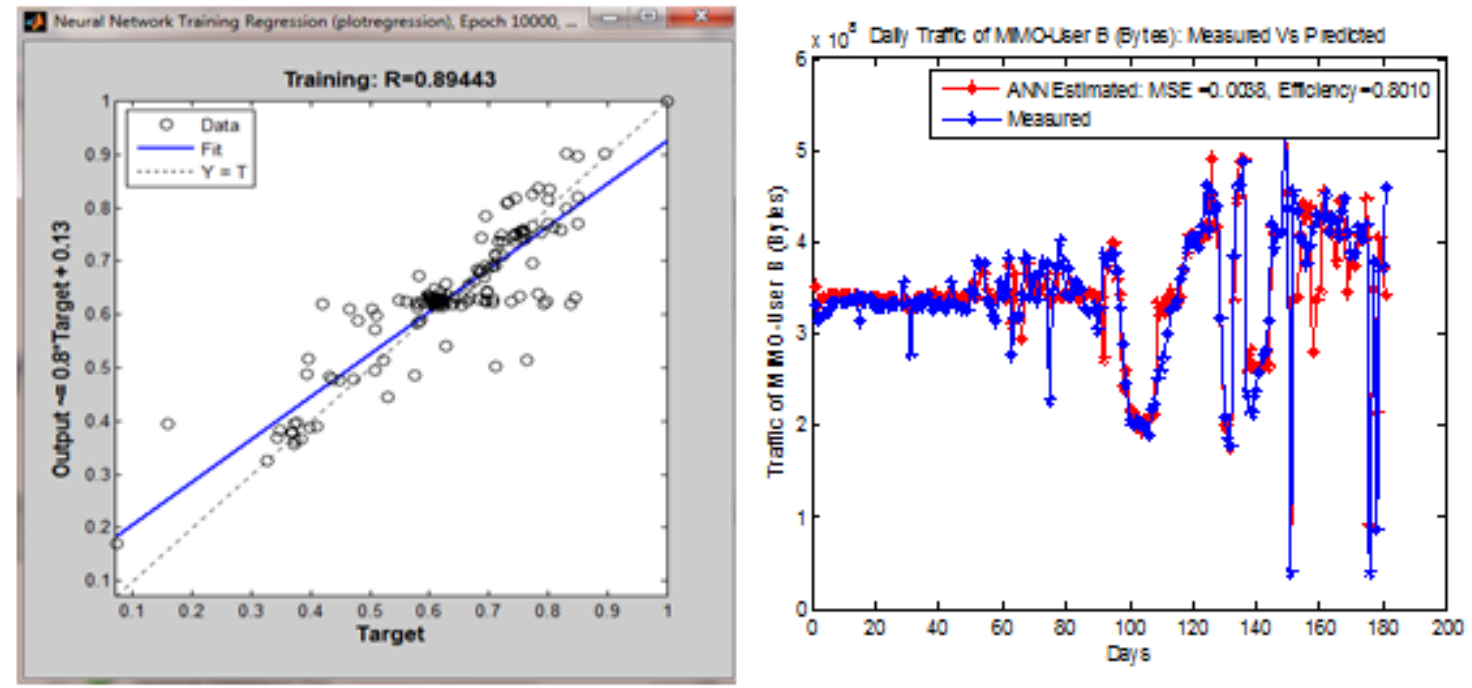

Figure 9. Results of modeling the daily Wimax traffic of MIMO-B users with (b) trainSCG

\section{E. Modeling results: daily WIMAX traffic of MIMO-AB users}

Figure 9 exhibits the result of modeling the WiMAX traffic for MIMO-AB users. Here, the graphical comparison for the prediction data against the actual data is plotted accordingly. It can be seen that there is a great fit between the two. This implies the high accuracy of the model in forecasting the WiMAX traffic data. 
International Journal of Artificial Intelligence \& Applications (IJAIA), Vol. 5, No. 5, September 2014

Table Iii Cross-Validation Report Of Ann Modeling Results Of Daily Wimax Traffic Of Mimo-Users Ab

\begin{tabular}{|l|c|c|c|c|}
\hline \multirow{2}{*}[\begin{array}{l|c|c|}{20}&{10}&{1}\end{array}]{} & \multicolumn{4}{|c|}{$T_{\text {Daily }(A B)}=f_{A B}\left(X_{\text {user }(\text { Max })}, X_{\text {user (Min })}\right)$} \\
\cline { 2 - 5 } & \multicolumn{3}{|c|}{ Training } & Testing \\
\hline Algorithms & MSE & $\mathrm{M}_{\text {eff }}$ & $\mathrm{R}$ & MSE \\
\hline TrainLM & 0.0009982 & 0.9998 & 0.96057 & $\mathbf{0 . 0 0 1 4}$ \\
\hline TrainSCG & 0.001538 & 0.9638 & 0.91877 & 0.0056 \\
\hline
\end{tabular}

The performance of two algorithms is compared and overall, TrainLM is superior among them. TrainLM shows the highest efficiency (0.9998) and lowest MSE (0.0014). On the other hand, the most inferior learning algorithm is TrainSCG with the highest MSE (0.0056) and lowest efficiency (0.9638). Here, it can be seen that the MSE of TrainSCG is very severe when compared to TrainLM. To understand the extent of severity, it is useful to note that the degree of error displayed by TrainSCG is four times as much as the one exhibited by TrainLM.
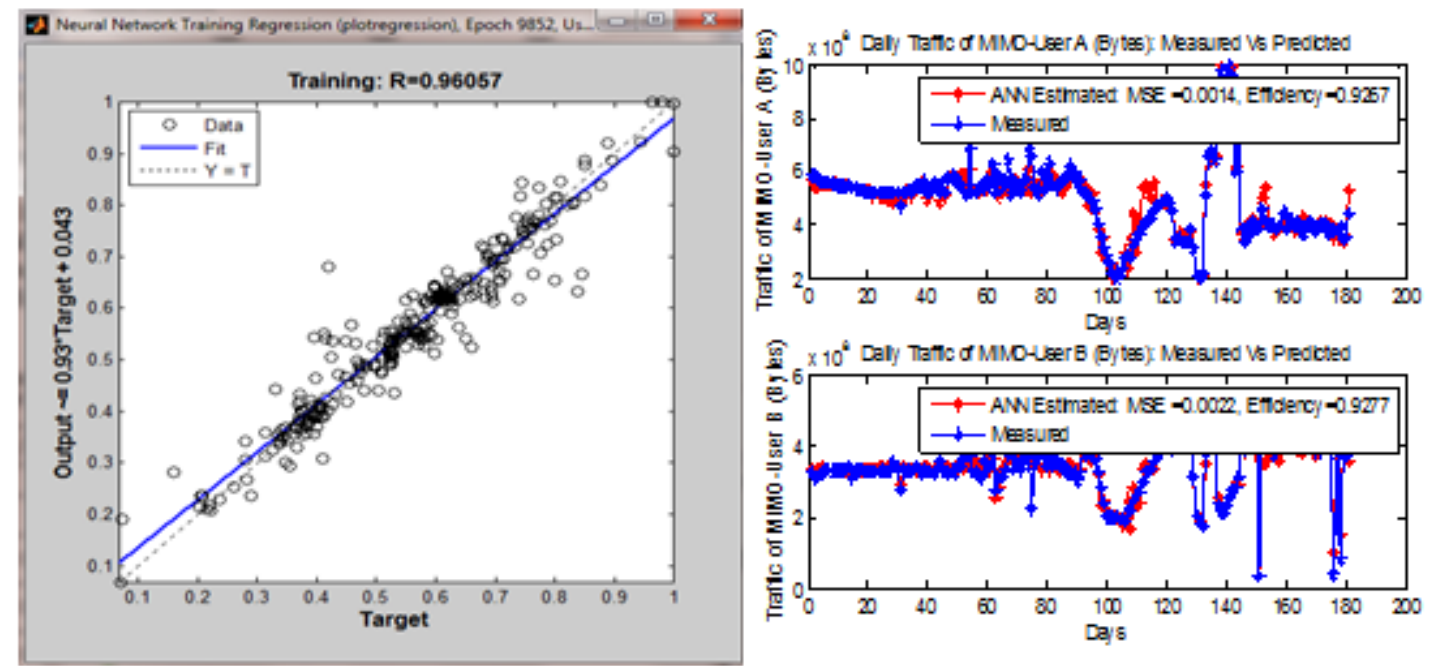

Figure 10. Results of modeling the dailyWimax traffic of MIMO-AB users with (a) trainLM 
International Journal of Artificial Intelligence \& Applications (IJAIA), Vol. 5, No. 5, September 2014
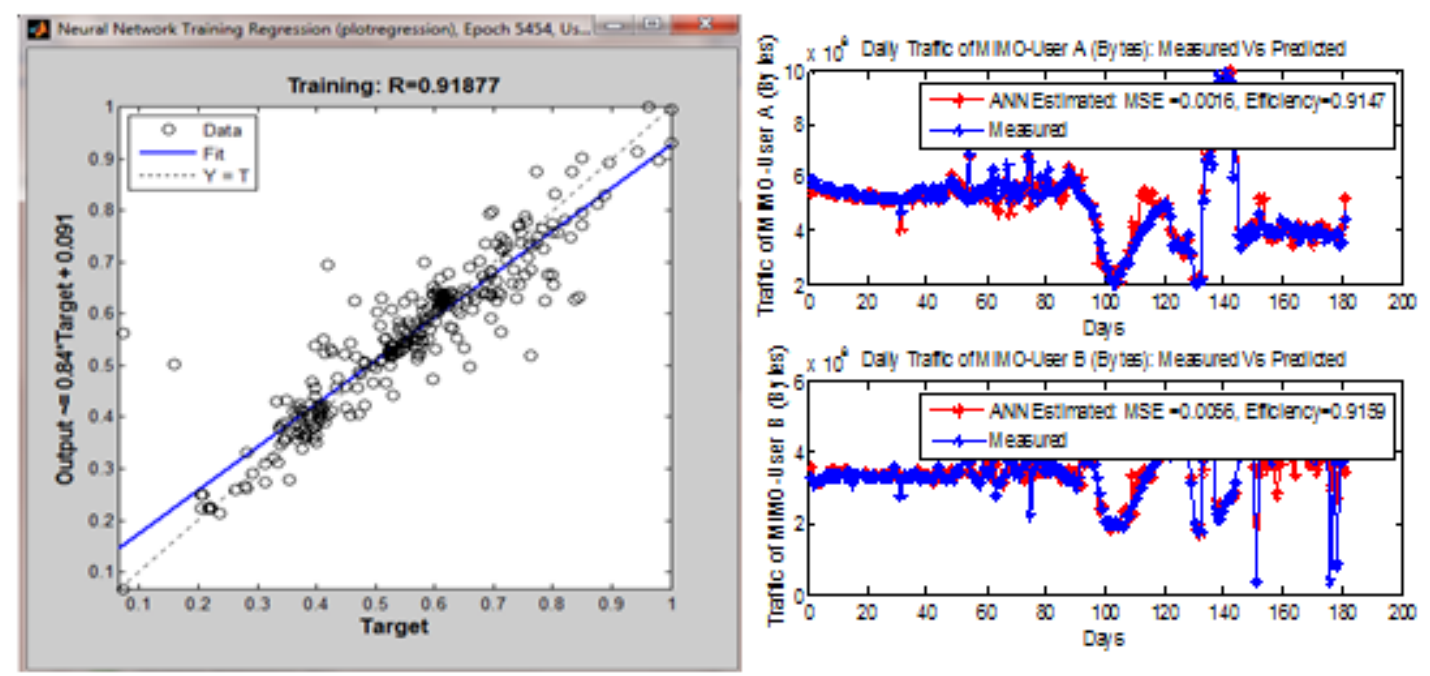

Figure 10. Results of modeling the dailyWimax traffic of MIMO-AB users with (b) trainSCG

\section{F. Modeling results: weekly WiMAX traffic of MIMO-A users}

From the weekly results of the MIMO-A users, it is quite apparent that TrainLM exhibits the best performance for the traffic forecasting with the lowest MSE (0.0007) and highest efficiency (0.95529). The performance of TrainSCG is comparatively worst, with a highest MSE $(0.0010)$ and lowest efficiency (0.95404). The difference of efficiency level between the two algorithms is quite marginal however, with a value of 0.00125 .

Cross-Validation Report Of Ann Modeling Results Of Weekly Wimax Traffic Of Mimo-Users A

\begin{tabular}{|c|c|c|c|c|}
\hline \multirow[t]{2}{*}[\begin{array}{llll}{2}&{0}&{10}&{1}\end{array}]{} & \multicolumn{4}{|c|}{$T_{\text {Weekly }(A)}=f_{A}\left(X_{\text {user }(\operatorname{Max})}, X_{u s e r(\operatorname{Min})}\right)$} \\
\hline & \multicolumn{3}{|c|}{ Training } & Testing \\
\hline Algorithms & MSE & $\mathrm{M}_{\mathrm{eff}}$ & $\mathrm{R}$ & MSE \\
\hline TrainLM & 0.00097138 & 0.95529 & 0.97112 & 0.0007 \\
\hline TrainSCG & 0.0099844 & 0.95404 & 0.97589 & 0.0010 \\
\hline
\end{tabular}


International Journal of Artificial Intelligence \& Applications (IJAIA), Vol. 5, No. 5, September 2014
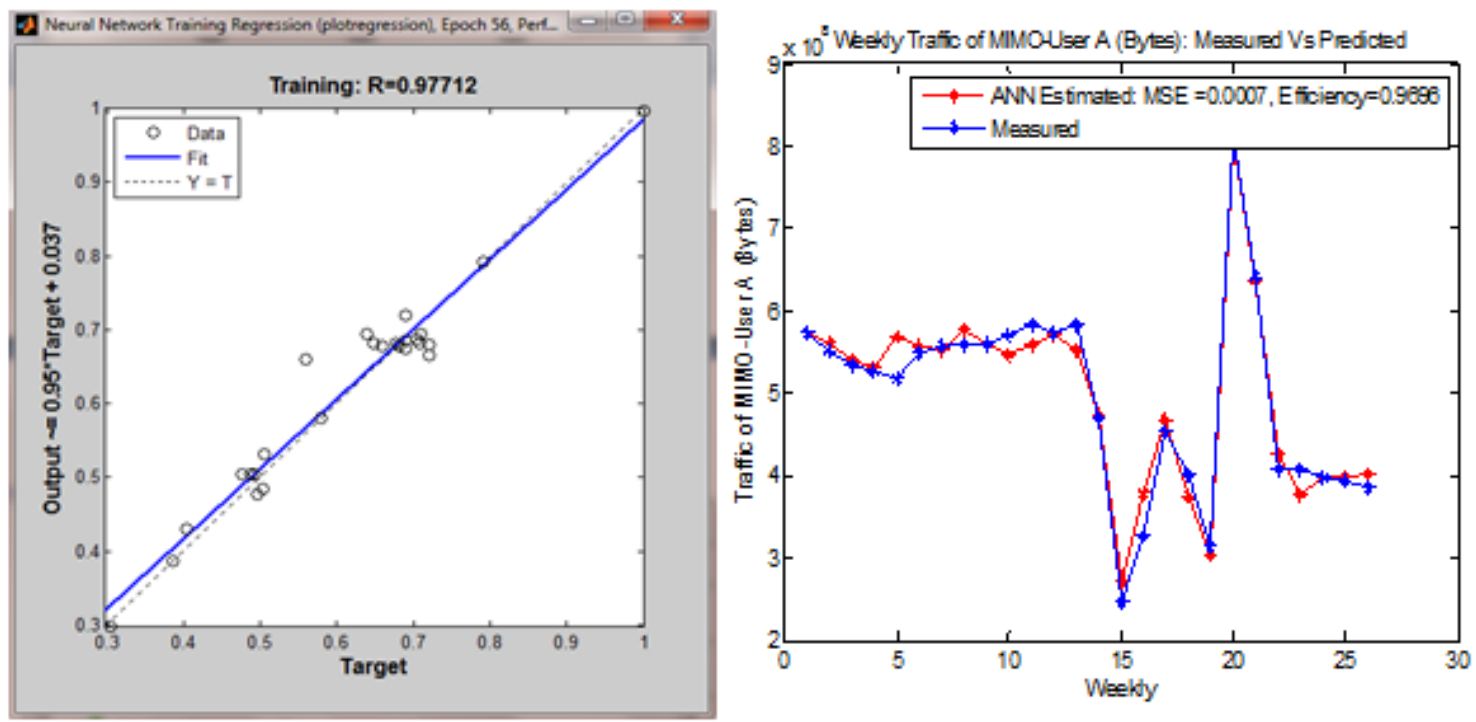

Figure 11. Results of modeling the weekly Wimax traffic of MIMO-A users with (a) trainLM
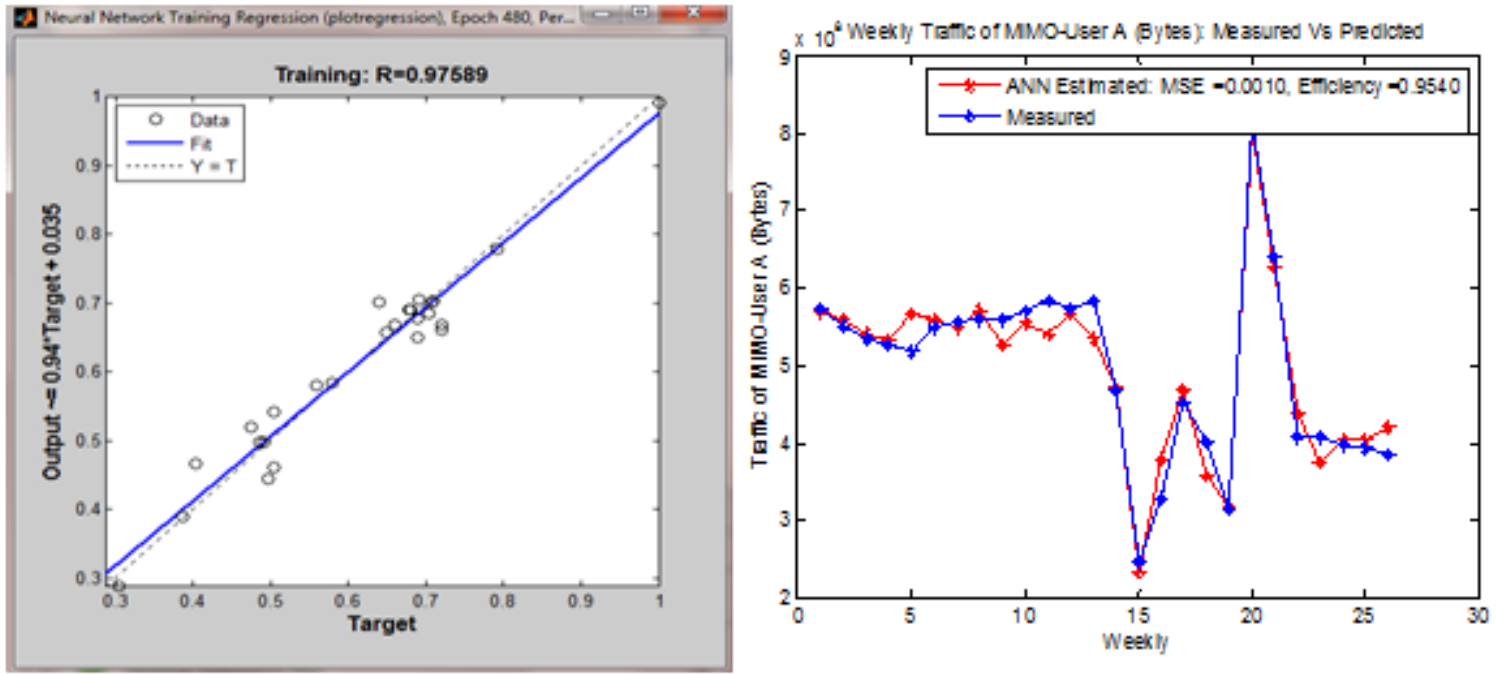

Figure 11. Results of modeling the weekly Wimax traffic of MIMO-A users with (b) trainSCG

\section{G. Modeling results: weekly WiMAX traffic of MIMO-B users}

For the results of the weekly traffic of MIMO-B users, the performance of both learning algorithms TrainLM and TrainSCG is similar in term of error with the same MSE (0.0010). However, TrainLM still outperforms TrainSCG in term of efficiency whereby the former achieves 0.95422 while the latter only manages an efficiency of 0.92209 . Here, the difference of efficiency is more significant when compared to the MIMO-A users. This indicates that the traffic behavior of MIMO-B users in training is more consistent when contrasted with MIMO-A users. 
International Journal of Artificial Intelligence \& Applications (IJAIA), Vol. 5, No. 5, September 2014

Cross-Validation Report Of Ann Modeling Results Of Weekly Wimax Traffic Of Mimo-Users A

\begin{tabular}{|l|c|c|c|c|}
\hline \multirow{2}{*}[20101]{} & \multicolumn{4}{|c|}{$T_{\text {Weekly }(B)}=f_{B}\left(X_{\text {user }(\operatorname{Max})}, X_{\text {user }(\text { Min })}\right)$} \\
\cline { 2 - 5 } & \multicolumn{3}{|c|}{ Training } & Testing \\
\hline Algorithms & MSE & $\mathrm{M}_{\mathrm{eff}}$ & $\mathrm{R}$ & MSE \\
\hline TrainLM & 0.00099665 & 0.95422 & 0.96067 & $\mathbf{0 . 0 0 1 0}$ \\
\hline TrainSCG & 0.009993 & 0.92209 & 0.96617 & 0.0010 \\
\hline
\end{tabular}
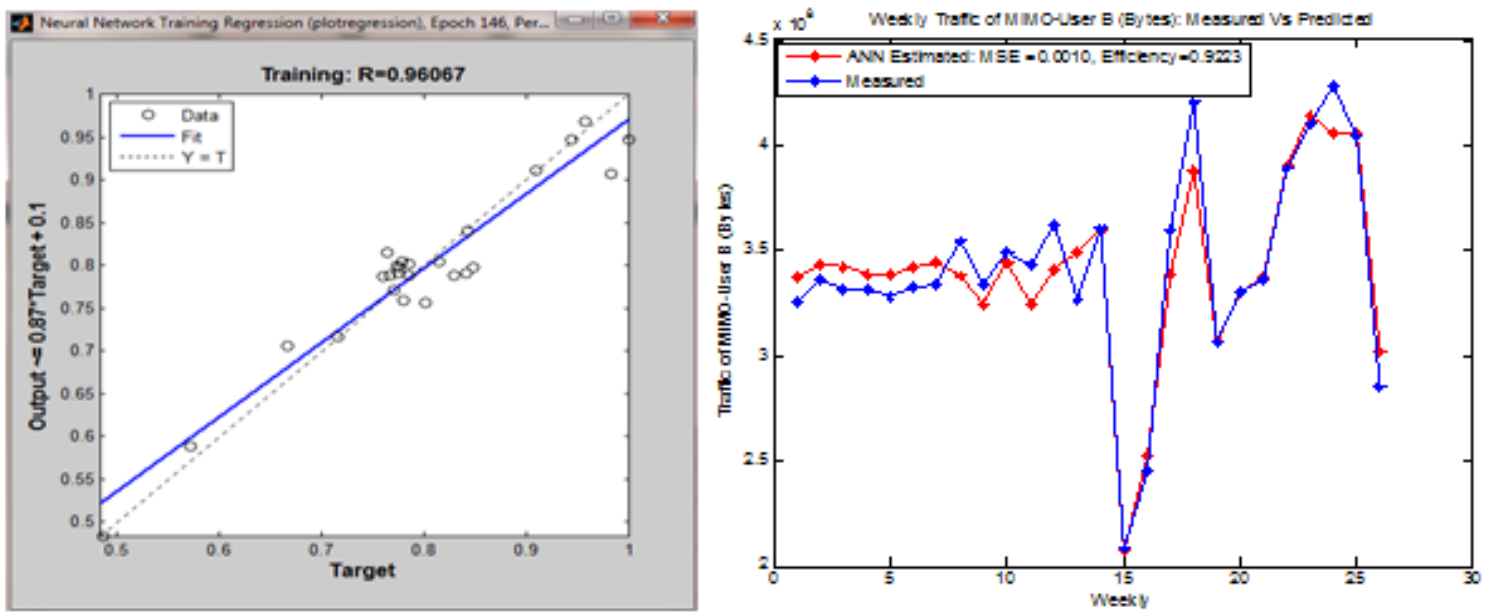

Figure 12. Results of modeling the weekly Wimax traffic of MIMO-B users with (a) trainLM
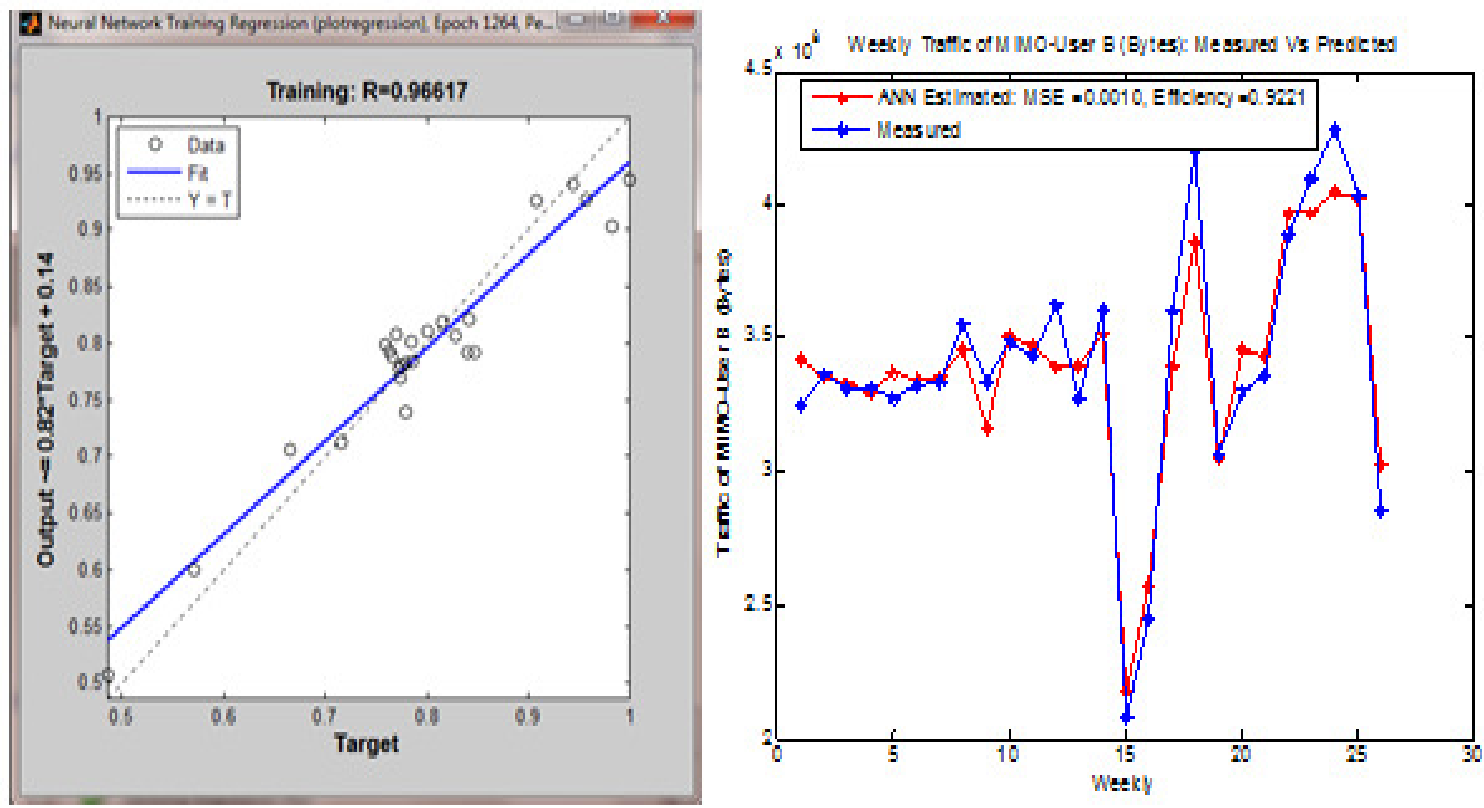

Figure 12. Results of modeling the weekly Wimax traffic of MIMO-B users with (b) trainSCG 


\section{H. Modeling results: weekly WiMAX traffic of MIMO-AB users}

Examining the results of the weekly MIMO-AB users, it is discovered that both learning algorithms show similar propensity in term of MSE (0.0010). However, the performance of TrainLM in term of efficiency (0.95422) is still better when contrasted against TrainSCG, which shows a significantly lower efficiency (0.92209). Overall, this implies that the weekly traffic of MIMO-AB users can be predicted more accurately with TrainLM instead of TrainSCG.

Cross-Validation Report Of Ann Modeling Results Of Weekly Wimax Traffic Of Mimo-Users Ab

\begin{tabular}{|c|c|c|c|c|}
\hline \multirow{3}{*}{\begin{tabular}{|c|} 
[20 10 1] \\
Algorithms
\end{tabular}} & \multicolumn{4}{|c|}{$T_{\text {Weekly }(A B)}=f_{A B}\left(X_{\text {user }(\operatorname{Max})}, X_{\text {user }(\operatorname{Min})}\right)$} \\
\hline & \multicolumn{3}{|c|}{ Training } & \multirow{2}{*}{$\begin{array}{c}\text { Testing } \\
\text { MSE }\end{array}$} \\
\hline & MSE & $\mathrm{M}_{\text {eff }}$ & $\mathrm{R}$ & \\
\hline TrainLM & 0.00099665 & 0.95422 & 0.96067 & $\mathbf{0 . 0 0 1 0}$ \\
\hline TrainSCG & 0.009993 & 0.92209 & 0.96617 & 0.0010 \\
\hline
\end{tabular}
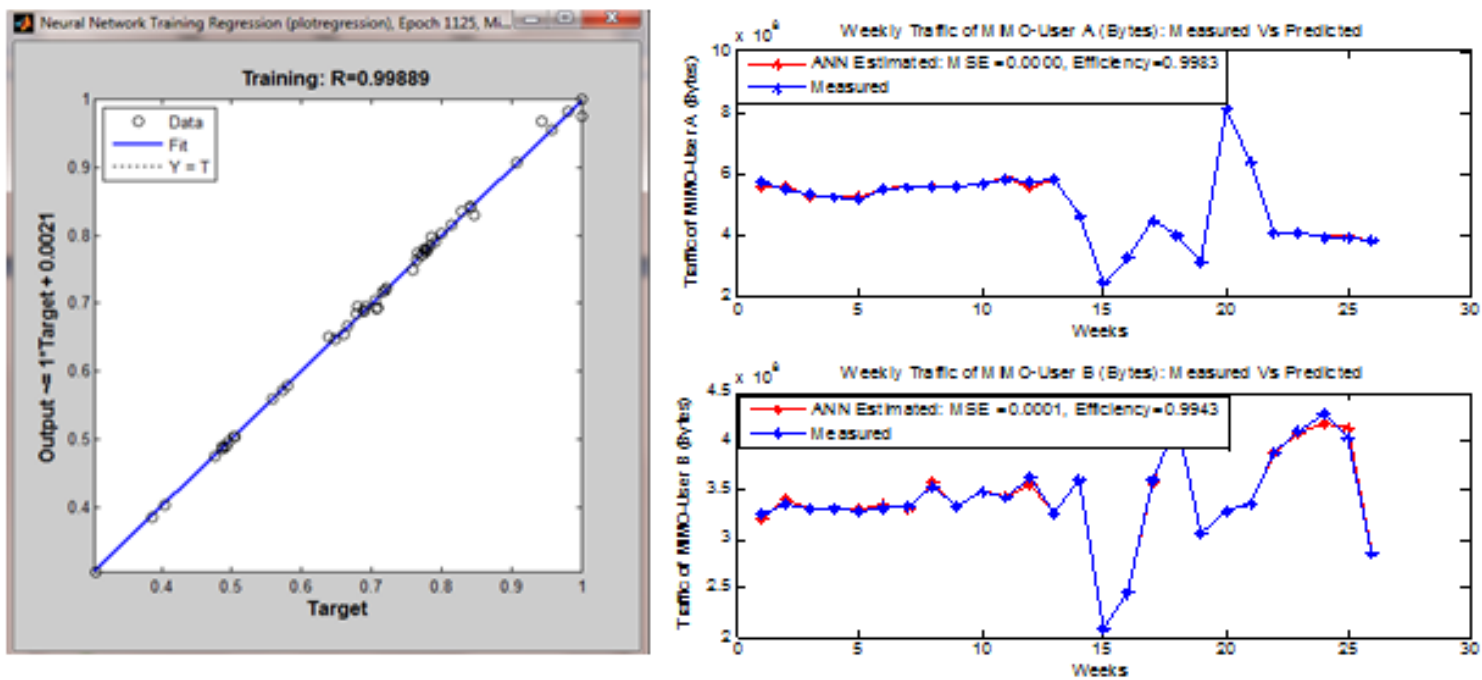

Figure 13. Results of modeling the weekly Wimax traffic of MIMO-AB users with (a) trainLM 
International Journal of Artificial Intelligence \& Applications (IJAIA), Vol. 5, No. 5, September 2014
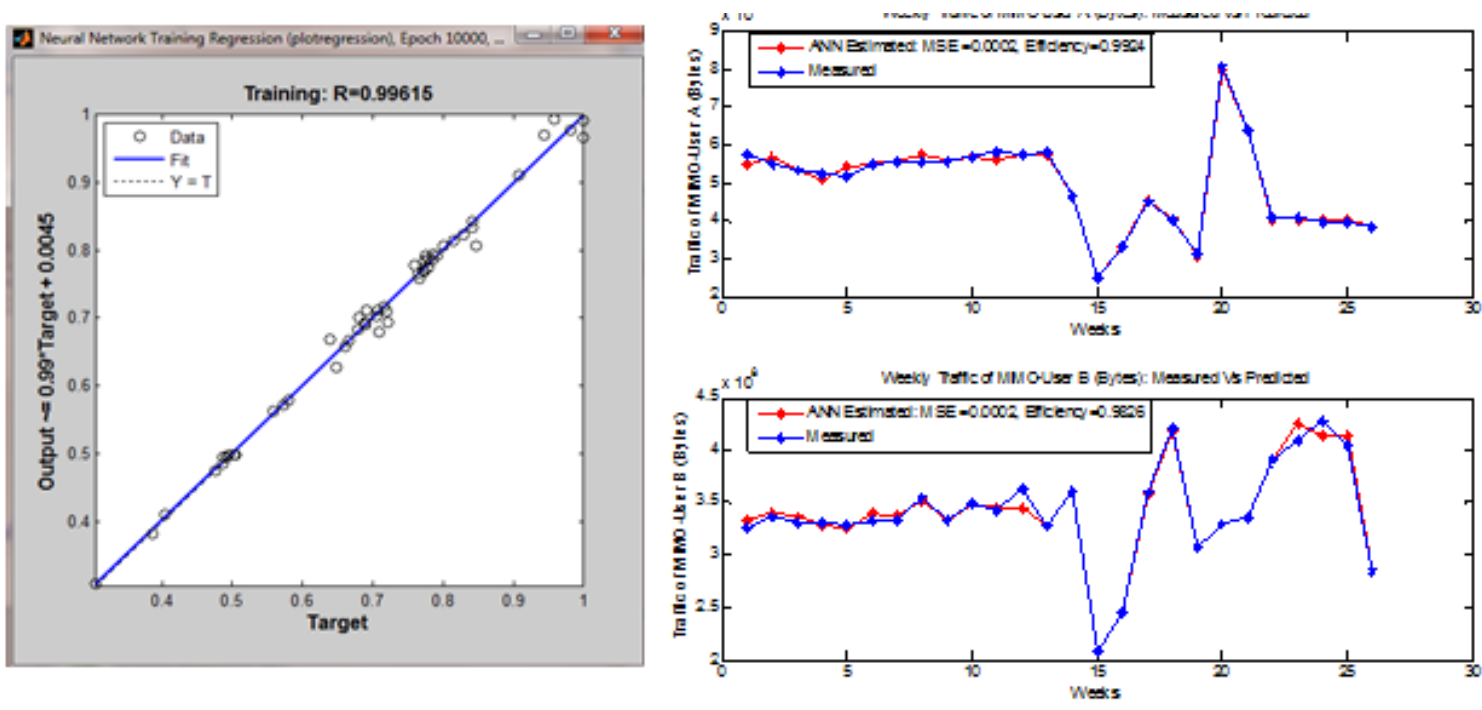

Figure 13. Results of modeling the weekly Wimax traffic of MIMO-AB users with (b) trainSCG

\section{CONCLUSION}

Forecasting the WiMAX traffic via the ANN model is a feasible approach in making prediction of the daily and weekly performance of the WiMAX network. It is possible to ascertain the traffic only by utilizing the maximum and minimum number of user online. Two algorithms, namely TrainLM and TrainSCG, are tested to determine the best model of forecasting. It is found that TrainLM, which invariably offers the lowest error and highest efficiency, shows the best performance.

\section{ACKNOWLEDGMENT}

The authors thank LibyaMax network (WiMAX technology) motorized by Libya Telecom and Technology for providing us the WiMAX traffic data and for helpful discussions around WiMAX network. We also thank staff of Faculty of Science and Technology, University Sains Islam Malaysia for their help and support.

\section{REFERENCES}

[1] Yang, Q. \& X. Wu, (2006) "10 challenging problems in data mining research", International Journal of Information Technology \& Decision Making 5(04): 597-604.

[2] Zhang, G., B. Eddy Patuwo \& M. Y Hu, (1998) " Forecasting with artificial neural networks: The state of the art", International journal of forecasting 14(1): 35-62.

[3] Adya, M., J. S. Armstrong, F. Collopy \& M. Kennedy, (2000) “ An application of rule-based forecasting to a situation lacking domain knowledge", International Journal of Forecasting 16(4): 477-484.

[4] Mitra, S. \& A. Mitra, (2006) “ Modeling exchange rates using wavelet decomposed genetic neural networks", Statistical Methodology 3(2): 103-124. 
International Journal of Artificial Intelligence \& Applications (IJAIA), Vol. 5, No. 5, September 2014

[5] Ibarra-Berastegi, G., A. Elias, R. Arias \& A. Barona, (2007) “ Artificial Neural Networks vs Linear Regression in a Fluid Mechanics and Chemical Modelling Problem: Elimination of Hydrogen Sulphide in a Lab-Scale Biofilter", Computer Systems and Applications, 2007. AICCSA'07. IEEE/ACS International Conference on, hlm. 584-587.

[6] Feng, H. \& Y. Shu, (2005) “ Study on network traffic prediction techniques", Wireless Communications, Networking and Mobile Computing, 2005. Proceedings. 2005 International Conference on, hlm. 1041-1044.

[7] Tan, C. N., (1993) “ Incorporating artificial neural networks into a rule-based financial trading system", Artificial Neural Networks and Expert Systems, 1993. Proceedings., First New Zealand International Two-Stream Conference on, hlm. 292-293.

[8] Taskaya-Temizel, T. \& M. C. Casey, (2005) " Configuration of neural networks for the analysis of seasonal time series. Dlm. (pnyt.). Ed”, Pattern Recognition and Data Mining hlm. 297-304. Springer.

[9] Clouse, D. S., C. L. Giles, B. G. Horne \& G. W. Cottrell (1997) “ Time-delay neural networks: Representation and induction of finite-state machines",Neural Networks, IEEE Transactions on 8(5): 1065-1070.

[10] Zhang, G. P. \& M. Qi, (2005) “ Neural network forecasting for seasonal and trend time series. European journal of operational research 160(2): 501-514.

[11] Daw, D. A. A. \& K. B., (2013 ) "Seman A Gateway To Wimax Profiling Services In Libya", International Journal of Engineering Research and Development. vol. 7, PP. 63-68.

[12] Railean, I., C. Stolojescu, S. Moga \& P. Lenca, (2010) " Wimax traffic forecasting based on neural networks in wavelet domain", Research Challenges in Information Science (RCIS), 2010 Fourth International Conference on, hlm. 443-452.

[13] I. Firoiu, C. Stolojescu, A. Isar, (2009) Forecasting of WiMAX BS Traffic: Observations and Initial Models, Alcatel Lucent technical report. 